\title{
Context in the Context of Physics and Learning
}

\author{
Noah D. Finkelstein \\ Department of Physics and Laboratory of Comparative Human Cognition \\ University of California, San Diego \\ nfinkels@ucsd.edu
}

This paper re-centers the discussion of student learning to focus on context. A theoreticallygrounded understanding of context and the relation of context to student learning are developed. This work argues for a contextual constructivist model of student learning, in order to support efforts in creating and analyzing environments that support student learning in physics.

\section{INTRODUCTION:}

To date, the discussion of student learning in physics has been largely student and content centered.[1] The common goal of many of these efforts is the creation of systems of activities which provide the opportunity for conceptual change in students who otherwise are left out by traditional forms of instruction. While researchers go to great lengths to create environments supportive of such activities, these environments, which promote student learning, remain under-theorized. The micro-cultures and contexts instantiated in institutional practice remain implicit in these approaches to student learning. If we are to understand what and how elements of learning environments are productively organized to shape students' understanding, we must develop a definition of these environments (contexts) and theories of how these contexts affect student learning. This paper articulates what is meant by context, and begins to develop a theory of how contexts shape (and are shaped by) student learning.

In a simplified summary of physics education research, scholars have moved from instructionist or transmissionist models to constructivist or interactive- engagement models of student learning. I propose moving the conversation further, to a contextual (or cultural) constructivist model, which places context in the middle of the discussion. Context is central to student learning, not as an analytically separate factor, not as the backdrop to student learning, but as an integral part of the learning process. Students (and other educational participants) shape and are shaped by the contexts in which these educational endeavors occur. Inherent in a given context are certain features which promote or inhibit construction of content understanding. From this perspective, conceptual change is mutually constituted by the individual and the context -- the central tenet of contextual constructivism.

\section{BACKGROUND AND EDUCATIONAL MODELS: \\ The classic "instructionist" or} "transmissionist" model for delivery of education idealizes the student as an empty vessel and instruction as the delivery of reified decontextualized knowledge. Redish describes this mode of instruction.[2] The student arrives at an instructional setting as an empty vessel and is "filled-up" with information by the instructor. The student then possesses the information and may call upon it as necessary. This model is a reasonable description of the practice of physics education found at the majority of high schools and introductory college level classrooms in the United States. It has long been criticized.[3]

Most current theories of student learning in physics, however, fall into the constructivist camp. The constructivist model focuses on the student as an active agent who constructs an understanding of content. Mestre describes this mode of learning as a lifelong, effortful process where a student's previously constructed understanding of the world is built upon during the learning process.[4] When students learn, they do not arrive as empty vessels, but rather, with some existing knowledge (about the world) which affects how they learn. The state of students' prior knowledge and the role that it serves has been the subject of some debate. On the one hand, a fair amount of work has been done on theorizing and cataloging students' misconceptions in order to develop strategies to confront and dispel these incorrect physical 
models. [5] On the other hand, others have spent time developing models to understand how students develop these naive or intuitive theories of the world in order that they may build on students' prior knowledge to construct a robust, scientific understanding of the world.[6]

Other models acknowledge that a variety of additional student characteristics affect learning.[7,8] A more thorough understanding of what is going on "inside" the student is critical to developing models of students' learning. Including a more thorough description of the student is what I call an enhanced constructivist view. ${ }^{1}$ One track of investigation examines students' own beliefs about what constitutes knowledge in the domain of physics. diSessa, Hammer and Elby have been studying student epistemology and how it affects learning.[7] They argue that theories of what it means to know and what constitutes knowledge in physics shape both student learning and instruction. For example, the curriculum that one develops and how a student performs (on certain measures of conceptual understanding of physics) depend upon how one views the nature of student knowledge.[8] These models are depicted graphically in figure 1 .

Implicitly acknowledged (and occasionally explicitly stated) is that these models depend upon context. I argue that to ignore the role of context in student learning is akin to ignoring the variability of the students themselves in the learning process. We no longer believe that

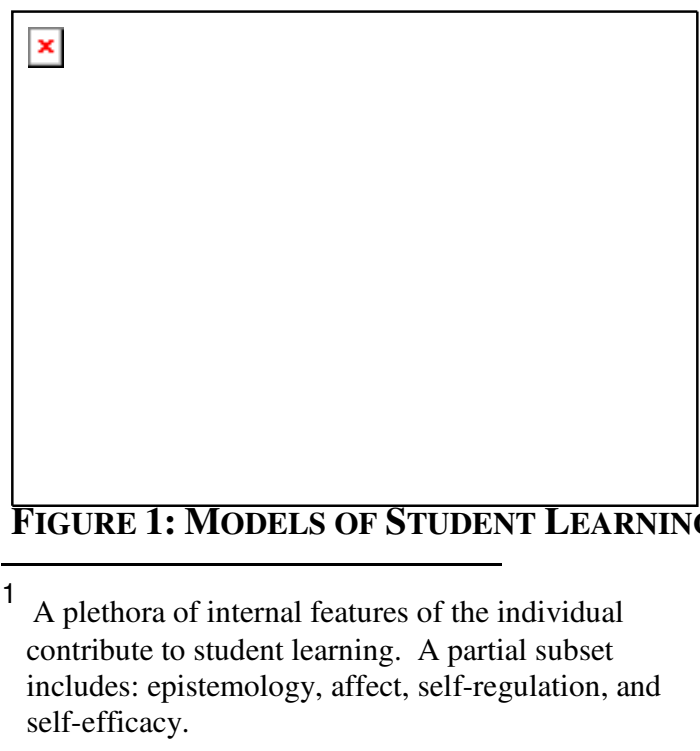

students are homogenous; they arrive with diverse understandings of the world and collected histories of experiences which shape their learning. We also know that the situation in which learning occurs and learning itself are related. A variety of research efforts in physics education have explicitly highlighted the importance of social, environmental, and contextual factors.[7,2] However, these issues are not the focal-point of discussion, but rather researchers often conclude their work by calling for attention to context. Context is still referred to casually without rigorous attention to its definition and to its explicit role in learning.

Moving context explicitly into the field of view when considering student learning is a core principle of contextual constructivism: students build an understanding of content in context and that context mediates student understanding of content. It is not possible to separate student learning from the context in which it occurs; Context is not a backdrop for student learning. Rather, context shapes student learning and is in turn shaped by both the content and student. This formation is depicted in figure 2. Context is as fundamental as the student and content. ${ }^{2}$

\section{CONTEXT ANDCONTEXTUAL CONSTRUCTIVISM:}

It is clear that the word context takes on a variety of meanings. In referenced pieces above, $[2,7,8]$ context has varied in meanings. It varies from the micro - how a specific problem is represented (word problem, pictorial or symbolic representation), or the setting of a problem (does it have to do with incline pulley, a raft and stream, or $\mathrm{x}, \mathrm{y}$, and $\mathbf{v}$ ?) to the macro - the macrocultural influences of various disciplines (math or physics) in western culture and what implications these have for student learning.

\footnotetext{
2 In a reductionist view of student learning and instructional paradigms, cognitive scientists [9] offer three instructional paradigms (behaviorist, developmental, and apprenticeship) which may be considered analogous to the those presented here (instructionist, constructivist, contextualconstructivist). However, contextual constructivism can not be reduced to a pure apprenticeship model, nor is it useful so strictly categorize instruction and student learning. Student learning in context includes elements of each of these paradigms (behavioral - incremental, developmental accommodation, and apprenticeship - acculturation).
} 


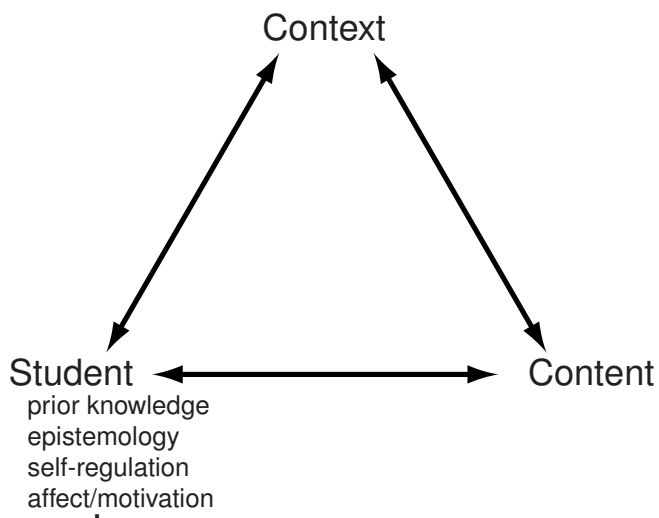

FIGURE 2: CONTEXTUAL CONSTRUCTIVIST MODEL OF LEARNING

While I argue that each of these different meanings is related and useful, and similar tools may be utilized in analysis of these varying notions of context, we must be more careful in distinguishing what we mean. Achieving a common reference is not a trivial problem, and I refer the interested reader to a book of papers devoted to analyzing what is meant by context.[10]

My development context borrows heavily from Michael Cole's work in the field of cultural psychology.[11] Cole emphasizes the importance of local and historical context, the language, environment, and tools of a community, and how these shape the process of learning.

Traditional definitions of context are: the local environment, or that into which things are placed, a setting.[12] In considering this definition of context we must be careful to avoid a static connotation, one in which the individual, content and the context in which they are embedded are analytically separate. McDermott warns against a static notion of context because it naturally leads to the view of the student as an empty vessel, and hence to the transmissionist model of student learning.[13] This is not to deny that there is use in the notion of context as that which surrounds. However, we must recognize that context arrives as an interaction between a learner and the local surroundings.

Another sense of context is useful to consider at this point. Cole returns to the Latin root of context, contexere, which may be translated as "to weave together" or "that which gives coherence to its parts.'[11] Birdwhistell's analogy of a rope is also useful.[13] A rope is made of individual fibers, none of which runs the length. The rope is not that which surrounds the fibers, rather it is the collection of fibers and relations of the fibers with each other -- the conditions of the system. Removing all the fibers from the rope and examining the rope and the fibers separately is not possible. Similarly, the task and its context are mutually constitutive. Learning and its context shape each other; neither may exist without the other.

It is useful to consider context as both embedded (that which surrounds) and as interactive (weaving together). As researchers begin to examine the role of context in student learning, the level of context to which we attend must be identified. Context may be embedded within context within context. Each interacts, influences and is influenced by adjacent levels of context. For example, we may consider three levels of context: the particular form a task takes (as in, the context of a particular problem whether the problem utilizes turntables or springs), the situation in which such action takes place, and the broader setting that creates the circumstances for the situation. I call these task formation, situation,[3] and idioculture,[14] respectively. ${ }^{3}$

To clarify with an example familiar to most physics educators, consider the contexts of a student learning about capacitance in a premedical physics sequence. The student engages in a task (solving a particular problem on capacitance) which is designed to facilitate student understanding of a concept (how capacitance adds in series or parallel). The presentation of the task is an example of task formation. The student, task (and even concept) exist within some broader activity, (e.g. doing problem sets). The activity, working with other students, is an example of a situation. Such a situation is part of a university class, a larger context, which is an example of idioculture. This idioculture is embedded in a university with

\footnotetext{
3 Here, situation is borrowed from Dewey. It is more than just the surrounding but the contextual whole to which experiences and objects are connected. Idioculture is borrowed from Fine. It is a "system of knowledge, beliefs, behaviors, and customs shared by members of an interacting group."[14]
} 
requirements, and so forth. For a given situation, such as working out a problem set for a given chapter, we must recognize that the activity is part of a larger class (e.g. E/M for biology majors) and that it includes certain tasks such as manipulating equations individually or in groups. Furthermore, student learning of capacitance is coupled to these embedded contexts. It differs from student learning of the 'same' content in a different set of contexts. Engineers for instance, may think of capacitors differently from premedical students. A pre-med student is likely to concentrate on deriving correct numerical values while an engineer may well focus on relevant applications.

\section{CONCluSIONS:}

Given these tools we may revisit the hypothesis of how context affects learning. An individual learns in context. Learning is not an isolated action, but rather a social activity influenced by the local contexts: task formation, situation, and idioculture. These contexts are not analytically separate, but integral to student learning. Inherent in a given context are certain allowances which promote or inhibit construction of content understanding. From this perspective, conceptual change is mutually constituted by the individual and the context. My goal is to create environments where the contexts support student learning of concepts. To do so, I propose it is essential to create environments with sufficient constraints and opportunities that students engaged in an activity become aligned with the concept at-hand. This alignment assures that the concept itself becomes meaningful and instrumental for the students. The concept becomes a tool for the task at-hand. Connected with the concept are the symbols and abstractions (e.g. equations or diagrammatic representations) necessary to allow the students to apply this concept to various other situations in useful manner -- i.e. which allow transfer. In short, the environment should support both the development of conceptual understanding in a particular situation as well as the ability to transfer such understanding to relevant new situations.

The author gratefully acknowledges support and assistance from LCHC colleagues, NSF funding (DGE\#9809496) and comments from the editors \& anonymous reviewers.

\section{REFERENCES:}

[1] McDermott, L.C. and Redish, E.F. (1999). Resource letter: PER-1: Physics education research. AJP, 67(9):755-767.

[2] Redish, E.F. (to appear 2002) Teaching Physics, New York: John Wiley and Sons;

Redish, E.F., (1999)"Millikan Award Lecture (1998): Building a Science of Teaching Physics," AJP, 67, 562-573

[3] Dewey, J. (1938) Experience and Education, New York: Collier Books

[4] Mestre, J. (1991) "Learning and instruction in pre-college physic science," Physics Today, 44(9), 56-62.

[5] Posner, G.J., Strike, K.A., Hewson, P.W., and Gertzog, W.A. (1982). "Accommodation of a Scientific Conception: Toward a Theory of Conceptual Change." Science Education, 66(2), 211-227.

[6] diSessa, A.A., (1988). Knowledge in Pieces, in Forman \& Puffall (Ed.) Constructivism in the Computer Age, Hillsdale NJ: Lawernce Erlbaum

[7] diSessa, A.A., Elby, A. \& Hammer, D. (to appear), J's epistemological stance and strategies, in G. Sinatra (ed.), Intentional Conceptual Change. Mahway, NJ: Erlbaum

[8] Hammer, D. (1996). "More than misconceptions: Multiple perspectives on student knowledge and reasoning, and an appropriate role for education research." AJP 64(10), pp. 1316-1325.

[9] Farnham-Diggory, S. (1994). "Paradigms of Knowledge and Instruction," Review of Educational Research, 64(3), 463-477.

[10] Chaiklin, S. and Lave, J., eds., (1993) Understanding Practice: Perspectives on Activity and Context. New York: Cambridge University Press.

[11] Cole, M., (1996). Cultural Psychology: a Once and Future Discipline. Cambridge, MA, Harvard University Press.

[12] Merriam-Webster On-Line Dictionary. http://www.webster.com/

[13] McDermott, R., (1993). "The acquisition of a child by a learning disability." in Understanding practice. Edited by S. Chaiklin and J. Lave, 269305. New York: Cambridge University Press.

[14] Fine, G.A.,(1987) With the Boys, Chicago: University of Chicago Press 